\title{
A New Approach to Diffraction Modelling for Line-of-Sight (LOS) Paths
}

\author{
S. Topcu ${ }^{1,2}$, P. Goktas ${ }^{1,3}$, E. Karasan ${ }^{3}$, and A. Altintas ${ }^{1,3}$
}

\begin{abstract}
In this paper, we present a new knife-edge diffraction method based on Bullington method for the terrestrial line-ofsight (LOS) paths. The diffraction loss of our new knife-edge diffraction method is compared with the results obtained from the Delta-Bullington method and the measurement data existing in the literature for sample fixed terrestrial microwave line-ofsight (LOS)/ non line-of-sight (NLOS) radio links. The revised Bullington method is shown to perform significantly better than the Delta-Bullington method for both overland and coastal/overwater radio links.
\end{abstract}

\section{INTRODUCTION}

Calculation of knife-edge diffraction loss is an important propagation issue in both mobile and fixed communication services. Terrestrial-path propagation modelling requires the prediction of the diffraction loss for a general path, that is, either line-of-sight (LOS) or non line-of-sight (NLOS). Diffraction theory indicates that the direct path between the transmitter and receiver needs a clearance above ground of at least $60 \%$ of the radius of the first Fresnel zone to achieve free-space propagation conditions. In such cases, the diffraction loss is assumed to be zero. However, if such a clearance is not satisfied, diffraction over the peaks of the obstacles in the signal path has to be considered. However, accurate determination of the diffraction loss is very challenging for realistic propagation environments such as mountainous regions. A common approximation is to use knifeedge that replaces the mountain peaks and ridges. The signal attenuation due to diffraction loss is an important constraint on the prediction of the path loss and the worst month link availability for the fixed terrestrial microwave LOS/NLOS radio links at frequencies of 2.0, 3.5, 5.0, 6.5 and $11.2 \mathrm{GHz}$.

Currently, a large number of propagation models predicting the excess loss due to terrain diffraction can be found in the literature. The most widely used approach to predict multiple knife-edge diffraction loss is to use an approximate method which simplifies the path geometry to calculate a total diffraction loss in terms of combinations of single-knife edge diffractions between adjacent edges over the LOS line. Some of them use the ideal knife-edge approximation of the obstacles between the transmitter (TX) and the receiver (RX), such as the Epstein-Peterson [1], Deygout [2], Bullington [3], Giovanelli [4] and DeltaBullington [5] methods, which can be described as geometrical techniques. The Deygout method [2] tends to overestimate the excess path loss when pairs of edges are very close to each other. In order to overcome this problem, an approximation correction derived from the exact analysis [7] of the two-edge solution over the LOS line was proposed by Causebrook [6]. Rec. ITU-R P.526 model [5] suggests Epstein-Peterson, Deygout and Delta-Bullington methods to calculate the diffraction loss due to multiple knife-edges. However, the Epstein-Peterson and Deygout methods are used only if there are two obstacles above the LOS line. Moreover, if all of the obstacles are below the LOS line, Rec. ITU-R P.526 model has provided only the Delta-Bullington method for calculating the diffraction loss. In this case, the DeltaBullington method is not based on constructing an equivalent hypothetical single knife-edge at the intersection of transmitter and receiver horizon rays.

This paper presents a new knife-edge diffraction method based on Bullington method [3] for the terrestrial microwave LOS radio links. The Bullington method for the calculation of the diffraction loss in NLOS path cases is extended for the LOS path cases [8]. This paper is organized as follows. The theory of our new knife-edge diffraction method is described in Section II. In Section III, the implementation of our knife-edge diffraction method is compared with both the Delta-Bullington method and the measurement data existing in the literature for sample fixed terrestrial microwave LOS/NLOS radio links [9], [10]. Concluding remarks are given in Section IV.

\section{THEORY OF PROPOSED KNIFE-EDGE DIFFRACTION METHOD}

Our knife-edge diffraction method is based on the Bullington construction for any type of path profile, whether LOS or trans-horizon. In the following equations, the distance, height and line-of-sight height of the $i$-th profile point are $d_{i}(\mathrm{~km}), h_{i}(\mathrm{~m}$, above sea level) and $l_{i}(\mathrm{~m})$, respectively and $i$ takes values from 1 to $n$ where $n$ is the number of path profile points. Transmitter and receiver heights are $h_{t x}$ and $h_{r x}$ in $\mathrm{m}$ (above sea level), and the complete path length is $d$ $(\mathrm{km})$. The effective Earth radius factor is given by $k$, and the Earth radius is taken as $6371 \mathrm{~km}$. The clearance of $i$-th profile point is given by:

$$
\text { clearance }_{i}=h_{i}+\text { Earth }_{-} l_{i}(\mathrm{~m})
$$

\footnotetext{
${ }^{1}$ Bilkent University, Communications and Spectrum Management Research Center (ISYAM), Ankara TR-06800, Turkey, e-mail: \{topcu, pgoktas, altintas\} @ ee.bilkent.edu.tr, tel.: +90 312 2902457, fax: +90 3122902410.

${ }^{2}$ Department of Computer Technology and Information Systems, Bilkent University, Ankara TR-06800, Turkey, e-mail: topcu@ee.bilkent.edu.tr, tel.: +90 312 2665907, fax: +90 3122665908.

${ }^{3}$ Department of Electrical and Electronics Engineering, Bilkent University, Ankara TR-06800, Turkey,

e-mail: \{pgoktas, ezhan, altintas\} @ee.bilkent.edu.tr, tel.: +90 312 2664307, fax: +90 3122664195.
} 
where

$$
\text { Earth }=1000 \times k R\left[\cos \left(\frac{d-d_{i}}{2 k R}\right)-\cos \left(\frac{d}{2 k R}\right)\right] \quad(\mathrm{m})
$$

Firstly, the dominant profile point with the highest slope of the line from the transmitter to the $i$-th profile point is given by:

$$
S_{\text {tim }}=\max \left[\frac{h_{i}+\text { Earth }-h_{t x}}{d_{i}}\right](\mathrm{m} / \mathrm{km})
$$

The slope of the LOS line from the transmitter to receiver site is also given by:

$$
S_{t r}=\frac{h_{r x}-h_{t x}}{d}(\mathrm{~m} / \mathrm{km})
$$

Two cases must now be considered.

$$
\text { Path Type }=\left\{\begin{array}{c}
L O S, \text { if } \mathrm{S}_{t i m} \leq S_{t r} \\
N L O S, \text { if } \mathrm{S}_{t i m}>S_{t r}
\end{array}\right.
$$

\section{Case 1. Path is LOS}

The highest slope of the line from the receiver to the $i$-th path profile point is given by:

$$
S_{\text {rim }}=\max \left[\frac{h_{i}+\text { Earth }-h_{r x}}{d-d_{i}}\right](\mathrm{m} / \mathrm{km})
$$

where the profile index $i$ takes values from 2 to $\mathrm{n}-1$.

One hypothetical knife-edge obstacle is defined by the intersection of $S_{\text {tim }}$ and $S_{\text {rim. }}$. The distance of an equivalent knife-edge obstacle from the transmitter is given by:

$$
d_{e q}=\frac{h_{r x}-h_{t x}+S_{r i m} d}{S_{t i m}+S_{r i m}}(\mathrm{~km})
$$

The clearance of an equivalent knife-edge obstacle is also given by the following formula:

$$
1 / h_{\text {clearance }}=\sum_{i=1}^{j} \frac{1}{\text { clearance }_{i}}
$$

where $j$ is the number of obstacles along the path profile.

Calculation of the Fresnel diffraction parameter, $v_{e q}$, for an equivalent knife-edge obstacle is given by:

$$
v_{\text {eq }}=h_{\text {clearance }} \sqrt{\frac{0.002 d}{\lambda d_{e q}\left(\mathrm{~d}-\mathrm{d}_{e q}\right)}}
$$

In that case, the diffraction loss of an equivalent knife-edge obstacle is calculated as follows [5].

$$
L_{\text {knife }}(\mathrm{dB})= \begin{cases}6.9+20 \log \left(\sqrt{\left(\mathrm{v}_{\text {eq }}-0.1\right)^{2}+1}+\mathrm{v}_{\text {eq }}-0.1\right), & \mathrm{v}_{\text {eq }}>-0.78 \\ 0, & \text { otherwise }\end{cases}
$$

\section{Case 2. Path is trans-horizon}

The intermediate profile point with the highest slope of the line from the receiver to the $i$-th path profile point is given by [5]:

$$
S_{r i m}=\max \left[\frac{h_{i}+500 C_{e} d_{i}\left(\mathrm{~d}-\mathrm{d}_{i}\right)-h_{r x}}{d-d_{i}}\right](\mathrm{m} / \mathrm{km})
$$

with effective Earth curvature $C_{e} \mathrm{~km}^{-1}$ is given by $1 / r_{e}$ where $r_{e}$ is the effective Earth radius in $\mathrm{km}$.
The distance of the Bullington point from the transmitter site is calculated by using Equation (6). Calculate the diffraction parameter, $v_{b}$, for the Bullington point [5]:

$$
v_{b}=\left[\mathrm{h}_{t x}+\mathrm{S}_{t i m} \mathrm{~d}_{e q}-\frac{h_{t x}\left(\mathrm{~d}-d_{e q}\right)+h_{r x} \mathrm{~d}_{e q}}{d}\right] \sqrt{\frac{0.002 d}{\lambda d_{e q}\left(\mathrm{~d}-\mathrm{d}_{e q}\right)}}
$$

In this case, the knife-edge diffraction loss for the Bullington point is given by:

$$
L_{\text {knife }}=J\left(\mathrm{v}_{b}\right)(\mathrm{dB})
$$

where the fuction $J$ is defined in [5].

For $L_{k n i f e}$ calculated using either Equation (9) or (12), Bullington diffraction loss with the correction term is now given by [5]:

$$
L_{b}=L_{\text {knife }}+\left[1-\exp \left(-\mathrm{L}_{\text {knife }} / 6\right)\right](10+0.02 \mathrm{~d})(\mathrm{dB})
$$

\section{EVALUATION OF PROPOSED KNIFE- EDGE DIFFRACTION METHOD}

Note that when the path type is LOS, we use our new method to calculate the diffraction loss. On the other hand, in Case 2, where the path is NLOS, we exactly use the diffraction loss calculation method defined in Rec. ITU-R P.526 [5]. The accuracy of our knife-edge diffraction method is justified by comparison with the diffraction loss predicted by Delta-Bullington method and the measurement data existing in the literature for sample terrestrial microwave LOS radio links. The short-term terrain diffraction measurements during August and September 2012 were performed in the area of Czech Republic mentioned in papers [9], [10]. These measurements were carried out for several combinations of transmitter and receiver heights at frequencies of 2.0, 3.5, 5.0, 6.5 and $11.2 \mathrm{GHz}$ for the case of both horizontal and vertical polarizations [9], [10]. In this paper, two different scenarios were examined. Detailed information regarding Scenario 1 is provided in Table 1. A path profile between transmitter and receiver sites is generated using Digital Terrain Elevation Data (DTED-1) with a spatial resolution of $3 \times 3$ seconds. The terrain path profile for different scenarios are shown in Fig. 1, 2 and 3 . The blue and red curves indicate the First Fresnel and 0.6 First Fresnel zone, respectively. Fig. 4 and 5 present the diffraction loss values for Scenario 1, obtained from the measurements, and calculated by the Delta-Bullington [3] and our knifeedge diffraction method. The path loss values for Scenario 2 obtained from measurements, and calculated by using the Delta-Bullington and our knife-edge diffraction method for the transmitter antenna height of $11.8 \mathrm{~m}$ are shown in Fig. 6. 


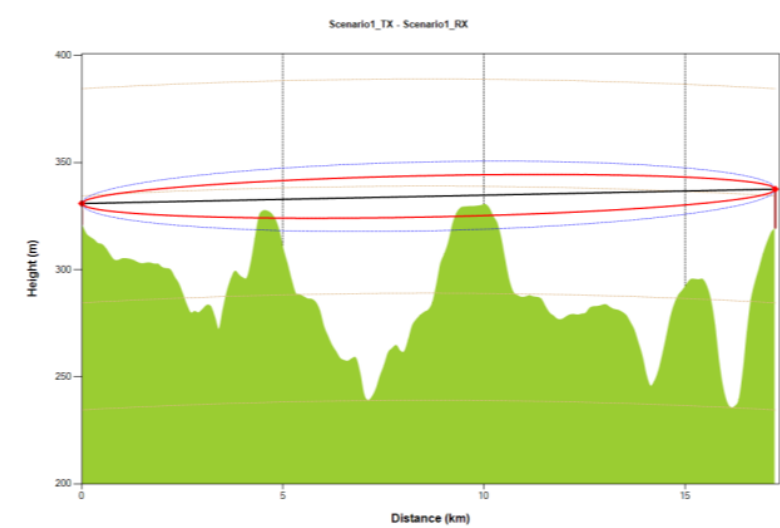

Figure 1. The terrain path profile for Scenario 1 (Frequency: $5 \mathrm{GHz}$, TX Antenna Height: $9.8 \mathrm{~m}$ a.g.l, and RX Antenna Height: 18.0 m a.g.1).

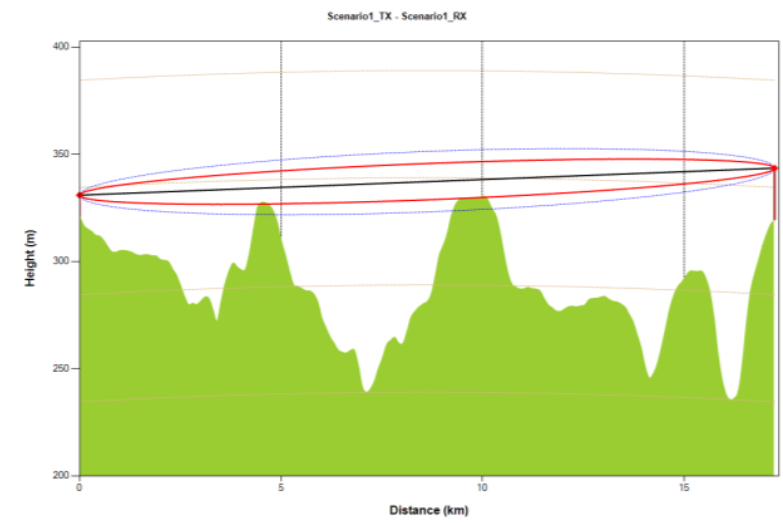

Figure 2. The terrain path profile for Scenario 1 (Frequency: 6.5 GHz, TX Antenna Height: 9.8 m a.g.l, and RX Antenna Height: $23.8 \mathrm{~m}$ a.g.l).

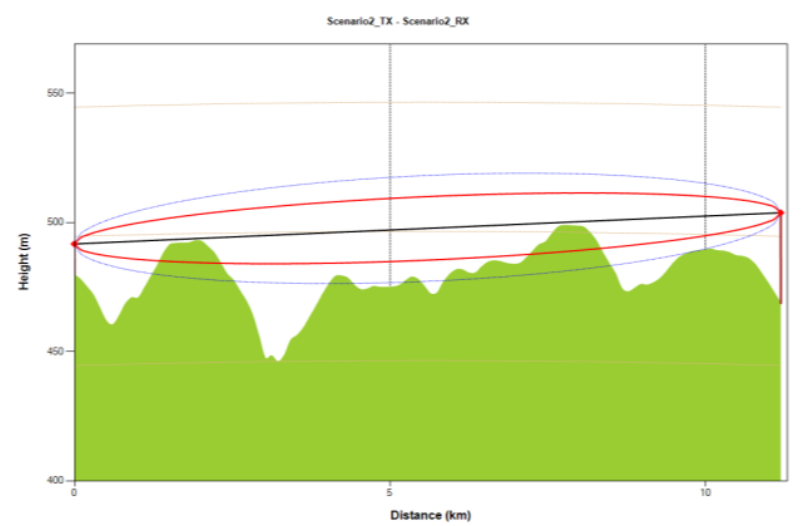

Figure 3. The terrain path profile for Scenario 2 (Frequency: $2 \mathrm{GHz}$, TX Antenna Height: $11.8 \mathrm{~m}$ a.g.l, and RX Antenna Height: 35.0 m a.g.l).

\begin{tabular}{|c|c|c|c|c|c|}
\hline Parameters & \multicolumn{5}{|c|}{ Scenario 1} \\
\hline Path length & \multicolumn{5}{|c|}{$17.3 \mathrm{~km}$} \\
\hline TX, latitude & \multicolumn{5}{|c|}{$50^{\circ} \mathrm{N} 13^{\prime} 56.13^{\prime \prime}$} \\
\hline TX, longitude & \multicolumn{5}{|c|}{$14^{\circ}$ E $01^{\prime} 59.22^{\prime \prime}$} \\
\hline $\mathrm{RX}$, latitude & \multicolumn{5}{|c|}{$50^{\circ} \mathrm{N} 10^{\prime} 53.36^{\prime \prime}$} \\
\hline RX, longitude & \multicolumn{5}{|c|}{$14^{\circ}$ E $15^{\prime} 43.54^{\prime \prime}$} \\
\hline $\begin{array}{l}\text { TX antenna } \\
\text { height (a.g.l) }\end{array}$ & \multicolumn{5}{|c|}{$9.8 \mathrm{~m}$} \\
\hline $\begin{array}{l}\text { RX antenna } \\
\text { height (a.g.l) }\end{array}$ & \multicolumn{5}{|c|}{$4.6-23.8 \mathrm{~m}$} \\
\hline $\begin{array}{l}\text { Radio frequency } \\
(\mathrm{GHz})\end{array}$ & 2.0 & 3.5 & 5.0 & 6.5 & 11.2 \\
\hline $\begin{array}{l}\text { Antenna gain } \\
(\mathrm{dBi})\end{array}$ & 9.8 & 11.7 & 11.4 & 10.7 & 35.0 \\
\hline $\begin{array}{l}\text { TX output power } \\
(\mathrm{dBm})\end{array}$ & 27.0 & 27.5 & 27.8 & 28.0 & 24.0 \\
\hline Polarization & \multicolumn{5}{|c|}{ Vertical or Horizontal } \\
\hline
\end{tabular}

Table 1. Terrestrial link and station parameters for Scenario 1.

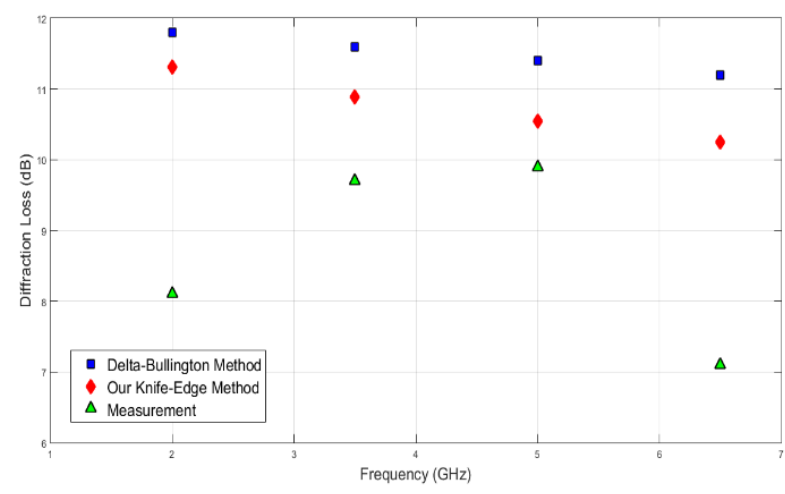

Figure 4. Path loss values for Scenario 1 obtained from measurements and calculated by using the DeltaBullington and our method for RX height of $18 \mathrm{~m}$.

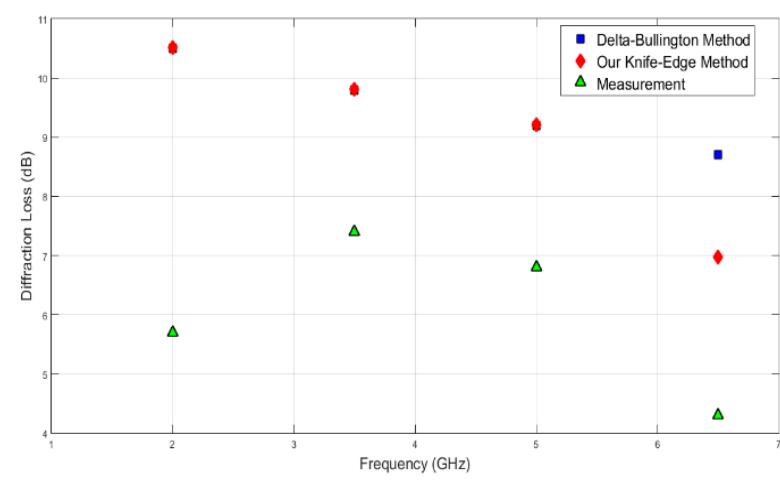

Figure 5. Path loss values for Scenario 1 obtained from measurements and calculated by using the DeltaBullington and our method for RX height of $23.8 \mathrm{~m}$. 


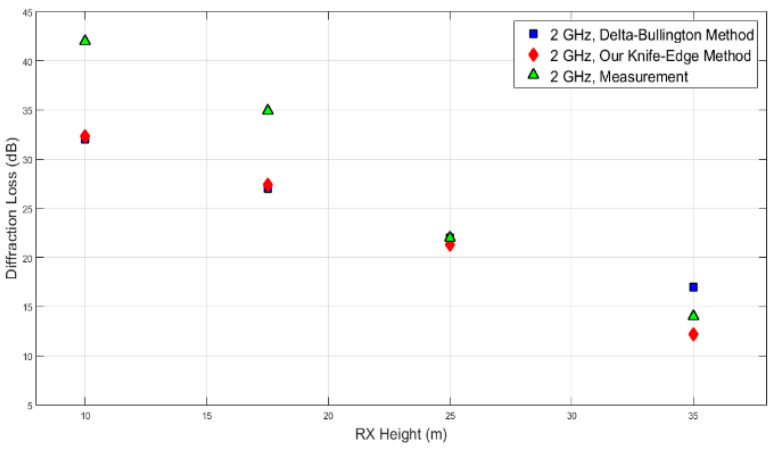

Figure 6. Path loss values for Scenario 2 obtained from measurements and calculated by using the DeltaBullington and our method for TX height of $11.8 \mathrm{~m}$.

The predictions obtained by using Delta-Bullington diffraction method are marked by squares, the path loss values calculated by using our knife-edge diffraction method are shown by circles, and the measurement data are marked by triangles. Based on the results presented in Fig. 4 and 5, the differences between measurement data and predictions produced by our method is small compared with the differences between measurements and predictions obtained by using Delta-Bullington method. Moreover, our knifeedge diffraction method satisfies $99.99 \%$ the worst month link availability criteria for Scenario 1 at frequencies of 2.0, 3.5, 5.0 and $6.5 \mathrm{GHz}$ [11].

In the case of NLOS path, a good match can be found between the predictions obtained by using the Delta-Bullington and our knife-edge diffraction method as shown in Figure 6. However, in the case of LOS path, the mean and standard deviation of discrepancies between measured and predicted loss values for our knife-edge diffraction method is small compared with the other prediction method for the case of RX antenna height of $35 \mathrm{~m}$, TX antenna height of $11.8 \mathrm{~m}$, and frequency of $2 \mathrm{GHz}$.

\section{CONCLUSIONS}

This paper presents a new knife-edge diffraction method based on Bullington construction for the LOS path. The performance analysis in terms of the excess loss due to terrain diffraction have been simulated and evaluated for the Delta-Bullington and our knife-edge diffraction method over sample terrestrial microwave LOS/NLOS radio links. Delta-Bullington [5] method significantly overestimates the loss values due to terrain diffraction for the sample LOS paths. On the other hand, our new knife-edge diffraction method performs considerably better and matches the measurement data available in the literature.

\section{References}

[1] J. Epstein and D. W. Peterson, "An experimental study of wave propagation at $850 \mathrm{Mc} / \mathrm{s}$," Proc. IEEE, vol. 41, pp. 595-611, May 1953.

[2] J. Deygout, "Multiple knife-edge diffraction of microwaves," IEEE Transactions on Antennas and Propagation, vol. 14, pp. 480-489, July 1966.

[3] K. Bullington, "Radio propagation at frequencies above 30 Megacycles," Proc. IEEE, vol. 35, pp. 1122-1136, October 1947.

[4] C. L. Giovanelli, “An analysis of simplified solutions for multiple knife-edge diffraction," IEEE Transactions on Antennas and Propagation, vol. 32, no. 3, pp. 297-301, March 1984.

[5] Recommendation ITU-R P.526, "Propagation by diffraction," International Telecommunication Union, Geneva, 2013.

[6] J. H. Causebrook and B. Davies, "Tropospheric radio wave propagation over irregular terrain: the computation of field strength for UHF broadcasting," BBC Research Report, 43, 1971.

[7] G. Millington, R. Hewitt, and F.S Immirzi, "Double knife-edge diffraction in field strength predictions," IEEE Monograph, 509E, 419-429, March 1962.

[8] P. Goktas, Analysis and implementation of prediction models for the design of fixed terrestrial point-to-point systems. MS thesis, Bilkent University, January 2015.

[9] M. Kvicera, et al., "Short-term terrain diffraction measurements: Preliminary results," in Proceedings of European Conference on Antenna and Propagation- $7^{\text {th }}$ Eur. Conf. Antennas Propag., Gothenburg, April 2013.

[10] M. Kvicera, et al., "Short-term diffraction measurements from L-to Q-Band: Results and Analysis," IEEE Transactions on Antennas and Propagation, vol. 62, no. 7, pp. 3693-3701, July 2014.

[11] P. Goktas, S. Topcu, E. Karasan and A. Altintas, "Multipath fading effect on terrestrial microwave LOS radio links," IEEE International Symposium on Antennas and Propagation and North American Radio Science Meeting, July 2015, Vancouver, BC. 